\title{
IMPACTO DE LA GESTIÓN DE RIESGOS EN LA PREVENCIÓN DE EVENTOS ADVERSOS DURANTE EL TRASLADO INTRAHOSPITALARIO DE PACIENTES ${ }^{1}$
}

\section{IMPACT OF RISK MANAGEMENT IN THE PREVENTION OF ADVERSE EVENTS DURING INPATIENT TRANSFER PATIENTS}

\author{
BlanCA RAMÍREZ ITURRA* \\ NALDY FEBRÉ ${ }^{* *}$
}

\begin{abstract}
RESUMEN
Durante su estadía, el paciente es sometido a muchos procedimientos que conllevan riesgos, incluyendo la actividad de traslado del paciente. La función del traslado cobra relevancia en cuanto a ocurrencia, si consideramos que se realiza a lo menos en 3 oportunidades durante su permanencia. En éstos, la condición del paciente puede ser auto-valente o de total minusvalía, como ocurre ante la ausencia de conciencia, presencia de vías de acceso venoso, catéteres u otros medios terapéuticos, factores que dan un grado mayor de complejidad a los movimientos interservicios y ponen en riesgo la ocurrencia de eventos adversos. Objetivo: Determinar el impacto de la gestión de riesgos en la prevención de eventos adversos o incidentes durante el traslado intrahospitalario de pacientes. Material y método: La presente investigación se define como cuantitativa, prospectiva con evaluación pre y postintervención, diseñada en 3 etapas: 1) Estudio de prevalencia de eventos adversos durante el traslado intrahospitalario; 2) Intervención a través de la aplicación de un Programa de Gestión de Riesgos; 3) Evaluación del impacto. Resultados: en la primera etapa fueron evaluados un total de 217 traslados, encontrando que en 123 casos $(56,7 \%)$ corresponde a eventos adversos relacionados a la "no confirmación de la identificación del paciente". Por lo que la intervención fue realizada en este evento. Después de la intervención la tasa de incumplimiento fue de 19.4\% ( $\mathrm{p}<0,000010$ ). Conclusiones: Los datos muestran que el programa de gestión de riesgos implementados impactó fuertemente en evitar el error postintervención.
\end{abstract}

Palabras clave: Transporte de pacientes, seguridad del paciente, eventos adversos, gestión de riesgos.

\begin{abstract}
In these, the patient's condition can be autonomous or totally disabled, as in the absence of consciousness, presence of venous access routes, catheters or other therapeutic methods, factors that give a greater degree of complexity to the inter movements, threatening occurrence of adverse events. Objective: To determine the impact of risk management in the prevention of adverse events or incidents during the intra-hospital transport of patients. Methods: This research is defined as quantitative, prospective pre and post intervention assessment,

${ }^{1}$ Trabajo presentado como prerrequisito para recibir el grado académico de Magíster en gestión de seguridad de pacientes y calidad de atención, Escuela de Enfermería, U. Mayor.

${ }^{*}$ Matrona, jefa de Operaciones Área Salud Facility Empresas Aramark. Magíster en gestión de seguridad de pacientes y calidad de Atención, dictado por la Escuela de Enfermería de la U. Mayor. Email: Brait2013@gmail.com

** Enfermera, Directora del programa de Magíster en gestión de seguridad de pacientes y calidad de atención, Escuela de Enfermería, U. Mayor. Email: nfebre@vtr.net
\end{abstract}


and designed in 3 steps: 1) Study of prevalence of adverse events during intra-hospital transport; 2) Intervention through the implementation of a Risk Management Program; 3) Evaluation of the impact. Results: In the first stage a total of 217 transfers were assessed, found that in 123 cases (56.7\%) is related to AE "no confirmation of patient identification." So the procedure was performed at this event. After intervention, the default rate was $19.4 \%(\mathrm{p}<0,000010)$. Conclusions: The data show that the risk management program implemented impacted strongly to avoid post intervention error.

Key words: Transportation of patients, patient safety, adverse events, risk management.

Fecha recepción: 21/01/14 Fecha aceptación: 26/03/15

\section{INTRODUCCIÓN}

Los sistemas de salud de todo el mundo reconocen la importancia que tiene entregar una atención segura y de calidad. Sin embargo, tanto la seguridad como la calidad no son temas exclusivos de los funcionarios que brindan atención directa, todo el entorno que rodea al paciente se involucra en esta visión. El paciente observa y asimila este trabajo desde su perspectiva, por ello es importante considerar que "las percepciones de los pacientes de la seguridad y el confort se correlacionan positivamente con la calificación técnica de trabajo" (1), evaluando permanentemente y reflejándose en los frecuentes cuestionamientos que lo pacientes hacen sobre la atención que se les brinda.

Unido a este enfoque y en plena era de la información, la calidad está centrada en el usuario, teniendo como una meta entender las necesidades de éste y luego satisfacer las demandas en los mercados. Éste concepto, derivado de los modelos industriales de gestión, enfatiza fundamentalmente la satisfacción del usuario que adquiere o hace uso del producto o servicio, que ha recibo por parte del centro de salud.

Por ende, la calidad de atención se resume en evaluar la satisfacción a través de algunos indicadores, tales como la existencia de seguridad en los distintos procesos, midiendo la ocurrencia de eventos adversos e incidentes durante la asistencia del paciente, aspecto que en su esencia siempre reviste gravedad, generando además en el paciente insatisfacción e inseguridad.

De acuerdo al marco conceptual de la clasificación internacional para la seguridad del paciente, publicada por la OMS, entenderemos por incidente "aquel suceso susceptible de provocar daño o conflicto en otras circunstancias, o que provoca la aparición de un evento adverso ligado por sobre todo a las condiciones de la asistencia”. Por su parte, evento adverso es definido como "todo hecho no deseado que ha causado daño al paciente o lo ha podido causar, ligado tanto a las condiciones de la asistencia, como a los del propio paciente y que nos e relacionan con el curso natural de la enfermedad" (2).

Desde esta mirada, el evento adverso (EA) recobra importancia, en la estrategia del Ministerio de Salud Chile (MINSAL), la que se refleja en el sistema de acreditación de prestadores, que incorpora, gran parte de los elementos de los procesos que se deben resguardar en beneficio de la calidad y seguridad de los pacientes (3).

La gestión de riesgos se define como "el conjunto de acciones que aunque no garanticen la ausencia de eventos adversos, tratan de agotar sus posibilidades de ocurrencia dentro de costos que sean sostenibles, es una metodología de trabajo que permite gestionar el 
riesgo al que sometemos a los pacientes con las atenciones médicas, para que éstos no se manifiesten al azar" (4).

La introducción de una estrategia de Gestión de Riesgos, orientada a crear un ambiente de trabajo basado en la confianza, enfocado al aprendizaje, es una manera efectiva de avanzar hacia el logro de prestar servicios de atención en salud de alta calidad (5).

La actividad de traslado intrahospitalario de pacientes forma parte de las actividades rutinarias que se desarrollan durante la hospitalización de éste, en el que participan diversos integrantes del equipo de salud, cumpliéndose protocolos establecidos (6). Los traslados hospitalarios de pacientes se realizan bajo condiciones de escasa o nula autovalencia, y son realizados por personal capacitado para tales fines; según la complejidad de la condición del paciente es el equipo que lo acompaña. Respecto a esta función diversos estudios destacan la necesidad que el personal involucrado en esta actividad "cuente con capacitación para ello y conozca los procedimientos correspondientes" $(7,8)$, en caso contrario se pueden desarrollar incidentes y/o eventos adversos, destacando los ocurridos por deficiencia en la gestión del traslado (falta de oxígeno, insumos, incumplimientos de protocolos como la identificación correcta de pacientes, entre otros).

Es por lo anteriormente expuesto, y dado que los procesos que involucran calidad y seguridad en la atención de los pacientes en el ámbito hospitalario son múltiples, que la presente investigación tiene como objetivo determinar el impacto en la prevención de incidentes y/o EA, durante el proceso de traslado intrahospitalario de pacientes, mediante la aplicación de un programa de gestión de riesgos. En base a este objetivo se planteó la siguiente hipótesis: las tasas de ocurrencia de incidentes y/o eventos adversos en la etapa preintervención es mayor a las tasas de ocurrencia de incidentes y/o eventos adversos en la etapa postintervención.

\section{MATERIAL Y MÉTODO}

Se efectuó una investigación de tipo cuantitativa prospectiva con evaluación pre y postintervención, diseñada en 3 etapas: En la primera (etapa preintervención) se seleccionó una institución de salud de gran tamaño docente asistencial, con un total de 450 camas, ubicada en Santiago de Chile. Para el cálculo de la muestra se consideró un universo de 560 traslados, obteniendo un total de 217 traslados evaluados, vigilados en forma prospectiva, en un periodo de 30 días (nivel de confianza de $95 \%$, error alfa de $5 \%$, valor esperado de 50\%). Se realizó un estudio de prevalencia de incidentes y/o EA durante el traslado intrahospitalario de pacientes, con el objetivo de determinar las tasas basales y las características epidemiológicas de éstos, los datos fueron registrados en ficha epidemiológica, creada para la presente investigación, validada por expertos y en campo; esta ficha epidemiológica fue estructurada en cinco partes: 1) datos generales del traslado; 2) datos relacionados a clasificación y gravedad del incidente o EA; 3 ) factores de riesgo predefinidos en tres criterios, los que se definieron por complejidad, donde el primer criterio se encontraba orientado a presencia de procedimientos invasivos, el segundo orientado a evaluar estado de conciencia del paciente y el tercero a la ocurrencia de eventos; 4) clasificación del momento de ocurrencia del Incidente o EA; 5) descripción breve del incidente o EA.

En la segunda etapa los datos fueron analizados, se seleccionó el incidente que presentó la mayor prevalencia de ocurrencia, el que correspondió a "No corroborar identidad del paciente"; con estos resultados se diseñó y aplicó la intervención basada en el programa de Gestión de Riesgos para la prevención de EA ocurridos durante el traslado, que consistió en análisis de los procesos relacionados al problema identificado, reestructuración de 
normativas, creación de los protocolos, capacitación del personal, elaboración de afiches recordatorios y definición del indicador de proceso para la evaluación del impacto (tasa de incumplimiento).

Luego del análisis inicial (diagnóstico), se implementó el programa de gestión de riesgos que consideró cambio en el sistema de vigilancia de pasiva a activa de los eventos adversos e incidentes relacionados al traslado; reestructuración del proceso de traslado, siendo incorporado un check list para asegurar que las actividades se desarrollaran, en especial la verificación de la identificación del paciente; educación y entrenamiento del personal encargado de esta actividad donde se incorporó metas internacionales de seguridad del paciente con énfasis en una identificación segura, el diagnóstico inicial y la importancia de la identificación correcta del paciente antes del traslado. Esta actividad se realizó en el $100 \%$ del equipo de traslado durante un mes. El programa de capacitación fue de tres horas presencial y una hora de capacitación en el lugar de trabajo. De acuerdo a planificación fueron realizadas mediciones de cumplimiento mensual, realizando medidas de mejora de acuerdo a los datos. Las evaluaciones para determinar el diagnóstico inicial y el impacto de la intervención fueron realizadas por las autoras de acuerdo a lo definido en protocolo.

Para la tercera etapa (periodo postintervención), de evaluación del impacto en la disminución de la tasa de incumplimiento, se realizó un estudio de prevalencia de Incidentes y/o EA orientada solo a incumplimiento de la corroboración de la identidad del paciente durante el traslado intrahospitalario, los datos fueron registrados en ficha epidemiológica ya aplicada en la primera etapa, ficha que se aplica prospectivamente en un total de 144 traslados en un periodo de 30 días.

Fueron respetados los principios éticos internacionalmente exigidos. La presente investigación fue aprobada por el comité de ética de la Facultad de Medicina de la Universidad Mayor, y aprobada por la institución en es- tudio; los funcionarios de traslado evaluados aprobaron la vigilancia realizada firmando el consentimiento informado. Los resultados fueron tabulados con el programa Stata 11.0, el análisis se realizó de acuerdo a comparación de tasas de ambas prevalencias realizadas para medir el impacto de la gestión de riesgos y su significancia estadística, mediante la tasa de incumplimiento. Se elaboraron tablas de frecuencia y se compararon los porcentajes de logro, utilizando el test de proporciones basadas en la distribución normal para comparar 2 proporciones, en este caso pre y postintervención, midiendo el nivel de significancia estadística utilizando el valor p para aceptar o rechazar la hipótesis nula.

\section{RESULTADOS}

Durante el periodo en estudio se cumplieron las actividades derivadas de las tres etapas, donde se elaboraron afiches de refuerzo, distribuyéndose en las áreas de trabajo. Se aplicó plan de capacitación al 100\% del personal de traslado, incluyendo jefaturas (79 funcionarios) y posteriormente se inició medición del impacto del programa sobre el evento detectado.

Los datos representados en la Tabla 1 muestran que la mayor frecuencia de presentación la ocupa "no corrobora identidad del paciente" correspondiendo a 123 casos, con $56.7 \%$ del total; sobre este Incidente se decide realizar la intervención.

En el periodo postintervención, la tasa de incumplimiento alcanza cifras de $19.4 \%$, se destaca que esta conducta después de la intervención aumenta de un 13.8 a un $80.6 \%$ de los casos revisados (144 traslados), al comparar ambas tasas la diferencia es estadísticamente significativa $(\mathrm{p}<0,00001)$, lo cual confirma la eficiencia de la intervención.

Los datos de la Tabla 2 muestran el nivel de cumplimiento de las conductas para una adecuada identificación de pacientes, pode- 
Tabla 1. Distribución porcentual de cumplimiento sobre el indicador "confirmación de la identidad del paciente en el proceso de traslado", para los periodos pre y postintervención, año 2013.

\begin{tabular}{lrrrc}
\hline \multirow{2}{*}{ Ítem evaluado } & \multicolumn{2}{c}{ Periodo } & \multicolumn{2}{c}{ Periodo } \\
& \multicolumn{2}{c}{ preintervención } & \multicolumn{2}{c}{ postintervención } \\
& No & $\mathbf{\%}$ & No & \% \\
\hline No confirma Identidad del Paciente & 123 & 56.7 & 28 & 19.4 \\
Confirma Identidad del Paciente & 30 & 13.8 & 116 & 80.6 \\
Otros ítemes & 64 & 29.5 & \multicolumn{2}{c}{$\mathrm{n} / \mathrm{r}^{*}$} \\
\hline Total & $\mathbf{2 1 7}$ & $\mathbf{1 0 0}$ & $\mathbf{1 4 4}$ & $\mathbf{1 0 0}$ \\
\hline
\end{tabular}

${ }^{*} \mathrm{n} / \mathrm{r}$ (no registrado)

Tabla 2. Porcentaje de cumplimiento de las distintas conductas relacionadas con la identificación del paciente durante el periodo postintervención ( $\mathrm{n}=144$ evaluaciones).

\begin{tabular}{lcc}
\hline Conducta & Número & $\begin{array}{c}\text { Porcentaje de } \\
\text { Cumplimiento }\end{array}$ \\
\hline Solo cumple con lectura del brazalete & 2 & 1,4 \\
Solo cumple con preguntar identidad al paciente & 2 & 1,4 \\
Solo verifica identidad con ficha clínica & 2 & 1,4 \\
Hace lectura del brazalete y verifica con ficha clínica & 44 & 30,6 \\
Consulta al paciente y verifica ficha clínica & 9 & 6,3 \\
Realiza las tres conductas de identificación del paciente & 57 & 39,6 \\
No realiza ninguna de las tres conductas de identificación & 28 & 19,4 \\
\hline Total & $\mathbf{1 4 4}$ & $\mathbf{1 0 0}$ \\
\hline
\end{tabular}

mos medir el nivel de cumplimiento de estas conductas relacionadas a la correcta identificación del paciente para el periodo postintervención; de esta tabla se puede concluir que un $80.6 \%$ de las personas capacitadas a través del programa de gestión de riesgo cumple con al menos una de las tres conductas exigidas en el protocolo de identificación del paciente. Si se considera que este porcentaje antes de la intervención era de un 13,8\%, se puede afirmar que la seguridad en la identificación del paciente durante el traslado ha aumentado significativamente $(\mathrm{p}<0,005)$.

La evaluación sobre el conocimiento de la normativa implementada sobre "identificación de pacientes durante el traslado" era conocida parcialmente por los funcionarios evaluados en el periodo preintervención. Si bien es obligatorio el uso de brazalete en todo ingreso de paciente, la lectura de éste para corroborar identificación era realizada solo por algunos auxiliares. Postintervención se logra que el 100,0\% tome conocimiento y aumente la aplicación de esta norma de seguridad.

Los datos de la Tabla 3 nos permiten evaluar que de 144 procedimientos de traslados evaluados, el 39\% de éstos cumplió el protocolo completo, esto es: 1) Lectura de brazalete. 2) Confirmación con el paciente y /o personal clínico. 3) Confirmación ficha clínica. Se destaca que el 19\% de los procedimientos evaluados no ejecuta adecuadamente la identificación del paciente previo traslado.

Se concluye que aplicado el programa de gestión de riesgo, un $80 \%$ de los traslados de pacientes realizado cumplió con el protocolo de revisión de identificación del paciente. 
Tabla 3. Cumplimiento de protocolo de identificación del paciente postintervención ( $\mathrm{n}=144$ traslados).

\begin{tabular}{lcc}
\hline Cumplimiento de protocolo & Número & Porcentaje \\
\hline Completo & 56 & 39 \\
Parcial & 59 & 41 \\
No cumple & 28 & 19 \\
Sin registro & 1 & 1 \\
\hline Total & $\mathbf{1 4 4}$ & $\mathbf{1 0 0}$ \\
\hline
\end{tabular}

\section{DISCUSIÓN Y CONCLUSIÓN}

El Instituto de Medicina de los Estados Unidos, en su informe "To err is human: Building a Safer Health Care" cuantificaron en los años noventa las muertes hospitalarias atribuibles a errores médicos prevenibles entre "al menos, 44.000 y quizás 98.000 personas en los Estados Unidos” (9).

Desde el pionero estudio "Harvard Medical Practice" dirigido por Brennan, las cifras oscilan entre un riesgo de padecer efectos adversos atribuibles a los cuidados de 2 por cada 100 hospitalizaciones; en el estudio de Utah y Colorado 3 al 16,6 por cada 100 hospitalizaciones $(10,11)$.

La OMS expresa que existe calidad de la atención de la salud, cuando en ella concurren un alto grado de excelencia profesional, el uso eficiente de los recursos, un mínimo de riesgos para el paciente, un alto grado de satisfacción del paciente, un impacto final positivo en la salud. Analiza la actitud frecuente del personal de salud que frente a la ocurrencia de eventos generalmente no reporta incidentes. Todo este conjunto de acciones ayuda a "no detener el daño al paciente. El protocolo de Londres establece errores de acción y errores de omisión" (8).

De acuerdo a la OPS, el error de ejecución es "la omisión de acciones que se deben realizar y están previstas en los procesos”(12). En la presente investigación en la etapa prein- tervención se pesquisa que el Error de ejecución sobre el ítem confirma la identidad del paciente previo al traslado se omite en un 56,7\%; de acuerdo a los autores, este incumplimiento se traduce en dos problemas de seguridad, siendo el primero clasificado como un incidente para el paciente trasladado erróneamente, el cual puede ser sometido a una intervención o procedimiento que no corresponde (donde correspondería notificar el evento adverso " procedimiento en paciente erróneo") y para el paciente que no se traslada, se puede notificar un evento adverso de "oportunidad de atención y retraso en el tratamiento, diagnóstico o cuidado".

En el año 2010 se publica una revisión sistemática que incluyó desde 1998 a 2009 eventos adversos con el objetivo de evaluar los eventos adversos durante el proceso de traslado instrahospitalario del paciente crítico; en este estudio los autores identificaron que los EA más frecuentes se relacionaron con reservas de oxígeno insuficientes, insuficiente conocimiento y la necesidad de mayor precisión de ajustes de respiradores y equipamiento, desconexión de tubos, alteraciones fisiológicas del paciente, entre otras; estos autores relatan incidencias de EA en estos procesos de traslados en rangos de un 5 a 70\%, concluyendo que éstos tienen relación con la "preparación y gestión como pasos cruciales, ya que tiene un impacto en el corto y mediano plazo en el pronóstico del paciente", consideran la iniciativa de organizar y preparar 
equipo humano y tecnológico previo al movimiento, para aminorar factores de riesgo (10). De acuerdo a estos autores la gestión es el paso "crucial", es por tal razón que en la presente investigación se seleccionó la metodología de gestión de riesgos, la que se inicia en la medición del riesgo (periodo preintervención) al que se encuentran expuestos los pacientes, donde encontramos que de 217 traslados evaluados, un importante número de pacientes trasladados presentaron eventos leves, con una tasa considerada por los autores de la presente investigación por sobre los definidos en la literatura analizada, esto puede deberse a la metodología aplicada, que permitió un seguimiento prospectivo de los eventos ocurridos. Los autores destacan que los traslados evaluados incluían pacientes críticos y no críticos (muestra no intencionada) y que el $100 \%$ de EA analizados fueron incidentes o eventos leves, al contrario de los referidos en la literatura.

De acuerdo a los datos de la literatura, la preparación del personal es un aspecto que disminuye la ocurrencia de factores de riesgo (13), esta visión debe ser complementada según Beckmann (11), con supervisión en el equipo de transporte $(14,15)$, lo anterior se refleja claramente en el programa de gestión de riesgo implementado en la presente investigación, programa que presentó un impacto en la reducción del riesgo en 95 casos.

En los traslados de pacientes críticos o en estado de riesgo, la morbilidad causada por esta actividad se considera "pobremente documentada y resalta la probabilidad de aumento en la duración de la hospitalización, secuelas neurosicológicas y mortalidad" (10). Es importante recordar en este punto, y dado el impacto de los incidentes o eventos adversos en proceso evaluados, las metas internacionales de seguridad del paciente, que definen la importancia de la identificación exacta del paciente para la realización de una acción, sea por personal médico o personal de apoyo $(10,16)$.

Es consenso internacional que la identi- ficación correcta de pacientes debe utilizar doble identificador (nombre completo y Rut o nombre completo y fecha de nacimiento) como mínimo, nunca utilizar abreviaturas, universalizar códigos, número de cama o habitación y confirmar la identificación del paciente previo a cada acción (17).

El presente trabajo muestra claramente que al iniciar la evaluación el proceso de traslado no era seguro para el paciente en un $100 \%$ pues no se aplicaba la doble verificación de la identidad; posterior a la intervención se puede concluir que el $80.6 \%$ de los pacientes trasladados deben estar seguros que fueron identificados correctamente.

Es importante considerar que para los autores este trabajo valida la aplicación de los programas de gestión de riesgo, siendo esta actividad de bajo costo y amplios beneficios para la calidad y seguridad de la atención.

En conclusión, la aplicación de un Programa de Gestión de Riesgo, muestra ser una metodología efectiva para el fomento de buenas prácticas en salud. Su aplicación es sencilla y de bajo costo, requiere solo de un trabajo sistemático y que permanezca en el tiempo. Como toda metodología, la medición permanente de su impacto debe formar parte del plan de acción.

Una de las principales limitaciones de este estudio deriva de la dificultad de eliminar el efecto Hawthorne sobre la observación directa relacionada con el cumplimiento del protocolo implementado para identificar correctamente al paciente previo traslado, lo que podría sobreestimar la frecuencia de los errores o de incidentes, pues los evaluados conocían previamente el objetivo de las supervisiones; sin embargo, pensamos que la intervención realizada con la metodología de gestión de riesgos logró identificar el problema, permitiendo una reestructuración del proceso y una posterior capacitación entre otras intervenciones que lograron un cambio de conducta que se traduce en el impacto en los resultados obtenidos. 
Agradecimiento: Los autores agradecen a la institución hospitalaria que permitió realizar el presente estudio de investigación y a la Empresa Aramark, quien se encuentra a cargo de entregar el servicio de traslado hospitalario en dicha institución, con vasta experiencia en este tema.

\section{REFERENCIAS}

1. Kjellberg K, Lagerström M, Hagberg M. Patient safety and comfort during transfers in relation to nurses' work technique. J Adv Nurs. 2004;47(3):251-9.

2. Organización Mundial de la Salud (OMS). El Marco Conceptual de la Clasificación Internacional para la seguridad del paciente [Internet]. Ginebra: OMS; 2009 [citado 2 junio 2014]. p. 1-160. Disponible en: http://www.who.int/patientsafety/implementation/icps/icps_full_report_es.pdf

3. Ministerio de Salud de Chile. Estrategia nacional de salud para el cumplimiento de los objetivos Sanitarios de la década 2011-2020 [Internet]. Santiago, Chile: Ministerio de Salud; 2011 [citado 2 junio 2013]. 426 p. Disponible en: http://web. minsal.cl/portal/url/item/c4034eddbc96ca6de0400101640159b8.pdf

4. Chomalí M, Febré N, Gattas S. La gestión de riesgo en el control de las infecciones de sitio operatorio. Rev. Med. Clínica Las Condes. 2007; 18(1):37-44.

5. Martínez López FJ, Ruiz Ortega JM, editores. Manual de gestión de riesgos sanitarios. Madrid: Díaz de Santos; 2001. 262 p.

6. Inga R. Estudio IBEAS. Estudio de Efectos Adversos en 58 Hospitales de Latinoamérica, 18vo Congreso Mundial de Epidemiología; 20-24 septiembre 2008; Porto Alegre, Brazil.

7. Lora J, García Sedeño MA. La satisfacción y la accesibilidad de los pacientes atendi- dos en el Servicio Provincial de Drogodependencias de Cádiz. (Sede Web) España. Trastornos Adictivos. 2007; 9(2): 132146.

8. Fajardo-Dolci G, Rodríguez-Suárez J, Campos-Castolo M, Carrillo-Jaimes A, Zavala-Suárez E, Aguirre-Gas H. Lineamientos Generales para el cuidado de la seguridad del paciente. Revista Conamed 2008; 13: 38-56.

9. Kohn LT, Corrigan JM, Donaldson MS. To Err is Human: Building a Safer Health System [Internet] Washington, D.C.: Institute of Medicine National Academy Press; 1999 [citado 2 junio 2013]. 8 p. Disponible en: http://www.iom.edu/ / media/Files/Report\%20Files/1999/ToErr-is-Human/To\%20Err\%20is\%20 Human \%201999\%20\%20report\%20 brief.pdf

10. Fanara B, Manzon C, Barbot O, Desmettre T, Capellier G. Recommendations for the intra-hospital transport of critically ill patients. Crit Care 2010; 14(3): R87.

11. Beckmann U, Gillies DM, Berenholtz SM, $\mathrm{Wu} \mathrm{AW}$, Pronovost P. Incidents relating to the intra-hospital transfer of critically ill patients. An analysis of the reports submitted to the Australian Incident Monitoring Study in Intensive Care. Intensive Care Med. 2004; 30(8):1579-85.

12. Organización Panamericana de la Salud. Sistemas de notificación de incidentes en America Latina [Internet] Washington, D.C.: OPS; Diciembre 2013 [citado 2 enero 2014]. 80 p. Disponible en: http://www.paho.org/hq/index.php?option $=$ com_content $\&$ view $=$ article $\&$ i $\mathrm{d}=8395 \% 3$ Asistemas-notificacion-incidentes-america-latina $\&$ catid $=1530 \% 3 \mathrm{~A}$ patient-safety\&Itemid=1557\&lang=es

13. Sánchez Vera Y, Corredor Grimaldos LH, Olivella González EO. Sistema de identificación, análisis y gestión de los eventos adversos en la ESE Hospital San Juan de Dios de Giron [Tesis Especialista]. Bucaramanga, Santander (Colombia): Univer- 
sidad CES y Universidad Autónoma de Bucaramanga; 2009.

14. Shirley PJ. Australia has considerable experience of transporting critically ill patients. BMJ. 1999; 319(7217):1137.

15. Shirley PJ, Stott SA. Intrahospital transport of critically ill patients. Anaesth Intensive Care. 2001;29(6):669.

16. Aranaz JM, Albar C, Galán A, Limón R,
Requena J, Alvarez EE, Gea MT. La asistencia sanitaria como factor de riesgo: los efectos adversos ligados a la práctica clínica. Gac Sanit. 2006; 20(Supl 1):41-7.

17. Damm C, Vandelet P, Petit J, Richard JC, Veber B, Bonmarchand G, et al. Complications during the intrahospital transport in critically ill patients. Ann Fr Anesth Reanim. 2005;24(1):24-30. 